\title{
Acute modulation of endothelial Akt/PKB activity alters nitric oxide-dependent vasomotor activity in vivo
}

\author{
Zhengyu Luo, ${ }^{1}$ Yasushi Fujio, ${ }^{1}$ Yasuko Kureishi, ${ }^{1}$ Radu Daniel Rudic, ${ }^{2}$ \\ Geraldine Daumerie, ${ }^{2}$ David Fulton, ${ }^{2}$ William C. Sessa, ${ }^{2}$ and Kenneth Walsh ${ }^{1,3}$ \\ ${ }^{1}$ Division of Cardiovascular Research, St. Elizabeth's Medical Center, Boston, Massachusetts, USA \\ 2Department of Pharmacology and Molecular Cardiobiology Program, Yale University School of Medicine, \\ New Haven, Connecticut, USA \\ ${ }^{3}$ Program in Cell, Molecular, and Developmental Biology, Tufts University School of Medicine, Boston, Massachusetts, USA \\ Address correspondence to: Kenneth Walsh, Division of Cardiovascular Research, St. Elizabeth's Medical Center, \\ 736 Cambridge Street, Boston, Massachusetts 02135, USA. Phone: (617) 562-7501; Fax: (617) 562-7506; \\ E-mail: kwalsh@opal.tufts.edu.
}

Received for publication January 19, 2000, and accepted in revised form July 14, 2000.

The serine/threonine protein kinase Akt (protein kinase B) phosphorylates endothelial cell nitric oxide synthase (eNOS) and enhances its ability to generate nitric oxide (NO). Because NO is an important regulator of vasomotor tone, we investigated whether Akt can regulate endotheliumdependent vasomotion in vivo using a rabbit femoral artery model of gene transfer. The endothelium of isolated femoral arteries was infected with replication-defective adenoviral constructs expressing $\beta$-galactosidase, constitutively-active Akt (myr-Akt), or dominant-negative Akt (dn-Akt). Femoral arteries transduced with myr-Akt showed a significant increase in resting diameter and blood flow, as assessed by angiography and Doppler flow measurements, respectively. L-NAME, an eNOS inhibitor, blocked myr-Akt-mediated vasodilatation. In contrast, endothelium-dependent vasodilatation in response to acetylcholine was attenuated in vessels transduced with dn-Akt, although these vessels showed normal responses to nitroglycerin, an endothelium-independent vasodilator. Similarly, relaxation of murine aorta ex vivo in response to acetylcholine, but not nitroglycerin, was inhibited by transduction of dn-Akt to the endothelium. These data provide evidence that Akt functions as key regulator of vasomotor tone in vivo.

J. Clin. Invest. 106:493-499 (2000).

\section{Introduction}

Nitric oxide (NO), produced by endothelial NO synthase (eNOS), is an important regulator of cardiovascular homeostasis. Endothelial-derived NO promotes vasodilation and inhibits platelet aggregation, leukocyte adherence, and vascular smooth muscle cell (VSMC) proliferation (1). These effects exert a profound influence on blood pressure, regional blood flow, vascular remodeling, and angiogenesis. The extent of eNOS activation, and thus NO release by the endothelium, is tightly regulated (2-4).

The serine-threonine protein kinase Akt, also referred to as protein kinase $\mathrm{B}(\mathrm{PKB})$, is a multifunctional regulator of glycogen synthesis, growth, and survival at a cellular level (5). Activation of Akt occurs through the binding of the lipid products of the phosphoinositide 3-kinase (PI 3-kinase) reaction to the pleckstrin homology domain of Akt, followed by translocation to the membrane and phosphorylation by PDK1 and PDK2 protein kinases. It is well established that activation of Akt can inhibit apoptotic death in many cell types in response to diverse stimuli (6-9). With regard to endothelial cells, Akt activation has been shown to be essential for cytoprotection induced by matrix attachment and VEGF $(10,11)$.

Recently, it has been recognized that exposure of endothelial cells to VEGF or physiological fluid shear stress stimulates NO production via the phosphoryla- tion of eNOS by Akt $(12,13)$. Mutation of the eNOS phosphorylation site (serine 1179) or expression of a dominant-negative version of Akt ( $\mathrm{dn}$-Akt) attenuates NO release in cultured endothelial cells. Recognition that Akt is a regulator of NO production suggests that the status of Akt activity in the vascular endothelium may also influence vasomotor tone and blood flow in vivo. Therefore, we examined whether Akt can regulate vasomotion and flow in the intact animal by using adenovirus-mediated transfer of dominant-negative and constitutively active Akt gene constructs to the endothelium.

\section{Methods}

Adenoviral constructs. Replication-defective adenovirus vectors expressing mouse Akt proteins fused in-frame to the hemagglutinin (HA) epitope under the control of the cytomegalovirus (CMV) promoter $(10,14)$. The dominant-negative mutant Adeno-Akt (Adeno-dnAkt) expressing the mutant Akt (T308A, S473A) construct cannot be activated by phosphorylation. The constitutively active Akt construct (Adeno-myr-Akt) has the c-src myristoylation sequence fused in frame to the $\mathrm{NH}_{2}$-terminus of the HA-Akt coding sequence. Adeno- $\beta$-galactosidase (Adeno- $\beta$-gal) has the $\beta$-galactosidase $(\beta$-gal) gene under the control of the CMV 
promoter (15). Viral titer was measured by standard plaque assay using 293 cells.

Rabbit femoral artery model. Thirty-six femoral arteries of male New Zealand white rabbits weighing 3.0-3.5 kg were analyzed in this study. The experimental protocol for this project was approved by the St. Elizabeth's Medical Center Institutional Animal Care and Use Committee and complied with NIH guidelines for care and use of laboratory animals. All rabbits were anesthetized with intramuscular ketamine $(50 \mathrm{mg} / \mathrm{kg})$ and xylazine $(5 \mathrm{mg} / \mathrm{kg})$. Femoral artery, popliteal artery, and saphenous artery were isolated. After temporary clamping of the proximal femoral artery and popliteal artery, either $100 \mu \mathrm{L}$ of saline alone, or saline plus Adeno- $\beta$ gal, Adeno-dn-Akt, or Adeno-myr-Akt, was infused into femoral artery via saphenous artery and incubated for 15 minutes. Viral titer was $1 \times 10^{10} \mathrm{pfu} / \mathrm{mL}$ for each experimental condition. After incubation, the saphenous artery was permanently ligated, and clamps on the proximal femoral artery and popliteal artery were removed to restore femoral blood flow. In the first experimental group ( $n=4$ vessels), animals were sacrificed 3 days after surgery and infection, and lac $Z$ expression in femoral arteries was analyzed as described later. In the second experimental group $(n=4$ for each treatment group: Adeno- $\beta$-gal, Adeno-dn-Akt, Adeno-myrAkt), femoral arteries were harvested 3 days after surgery and transfection for the measurement of cGMP levels. In the third experimental group $(n=5-8$ vessels for each treatment group: saline, Adeno- $\beta$-gal, Adenodn-Akt, Adeno-myr-Akt), femoral artery diameter was assessed by quantitative angiography, and blood flow was measured by intravascular Doppler analysis.

Angiography. Base-line vessel diameters and changes in vessel diameters in response to the endotheliumdependent vasodilator acetylcholine (Ach), the endothelium-independent vasodilator nitroglycerin (NTG), the NO inhibitor N $\omega$-nitro-L-arginine methyl ester (L-NAME) or papavarine were evaluated by angiography 3 days after surgery and adenovirus-mediated gene transfer. A 3F catheter was introduced into the left common carotid artery through a small cutdown and advanced to the proximal end of femoral artery with a 0.014 -inch guide wire (Hi-Torque Floppy II; Advanced Cardiovascular Systems, Temecula, California, USA). Contrast media (MD-76, meglumine diatrizoate and sodium) was then injected using an automated angiographic injector at a rate of $1 \mathrm{~mL} / \mathrm{s}$. Serial images of femoral artery were recorded at base line and immediately after administration of the different drugs, with a 5-minute interval between each drug administration (16). Drugs were administered by intravenous injection via a Tracker-18 infusion catheter (Target Therapeutics, Fremont, California, USA) in the following order: Ach, L-NAME immediately followed by Ach, and finally NTG, with angiographic assessment before and after each administration. The papavarine administration was performed on a second group of animals. Ach and L-NAME were administered at con- centrations of $5 \mu \mathrm{g} / \mathrm{kg} / \mathrm{min}$ for 2 minutes or a single bolus infusion of $20 \mathrm{mg} / \mathrm{kg}$, respectively. Papavarine was administered by a single bolus infusion of $2.0 \mathrm{mg}$. Fresh stock solutions were prepared immediately before each experiment. NTG was administered by a single bolus infusion of $200 \mu \mathrm{g}$.

Intravascular Doppler flow. Intravascular Doppler flow measurements in the femoral artery were performed immediately after each angiographic measurement using a 0.018-inch Doppler guide wire (Cardiometrics Inc., Mountain View, California, USA). The wire was advanced through the $3 \mathrm{~F}$ infusion catheter to the proximal segment of the femoral artery. A $12-\mathrm{MHz}$ piezoelectric transducer is mounted at the tip of the distal segment of the wire, allowing real-time spectral analysis of the Doppler signal, from which the average peak velocity (APV) is calculated and displayed on line. The luminal diameters of the femoral arteries were determined previously from records of serial angiographic images. Doppler scanning-derived flow was calculated as $\mathrm{Q}_{\mathrm{D}}=\left(\mathrm{pd}^{2} / 4\right)(0.5 \times \mathrm{APV})$, where $\mathrm{Q}_{\mathrm{D}}$ is Doppler-derived time average flow $(\mathrm{mL} / \mathrm{min}), \mathrm{d}$ is vessel diameter, and APV is time average of the spectral peak velocity (17). The mean velocity was estimated as $0.5 \times$ APV by assuming a time-averaged parabolic velocity profile across the vessel. Measurements were performed at rest and after administration of each drug and angiography.

Measurement of cGMP levels. Femoral arteries were harvested 3 days after surgery and transfection and cut into rings. Rings were immersed immediately in a solution of 3-isobutyl-L-methylxanthine (IBMX; 1 $\mathrm{mmol} / \mathrm{L}$ ) and incubated at $37^{\circ} \mathrm{C}$ for 30 minutes before being snap-frozen in liquid nitrogen and stored at $-70^{\circ} \mathrm{C}$ until the time of assay. cGMP levels were determined by a RIA kit (Amersham Pharmacia Biotech, Piscataway, New Jersey, USA) as described previously (18). Four vessel rings from four individual rabbits were examined per experimental group.

In vitro analysis of vascular function from adenovirusinfected mouse aortae. Four- to 6-week-old C57Bl/6J mice were anesthetized with ketamine/xylazine, and midline thoracotomy was performed. Mice were exsanguinated via transection of the abdominal aorta followed by perfusion with saline through the left ventricle. The heart and lungs were removed, exposing the thoracic aorta, and loose ligatures were placed on the ascending arch and the distal thoracic aorta. Adenoviral constructs encoding $\beta$-gal and dn-Akt were delivered in a retrograde manner $\left(4.5 \times 10^{11}\right.$ particles per milliliter; $20 \mu \mathrm{L}$ ) until the aorta was filled. Sutures were then tightened, and the aorta was left in situ for 15 minutes at $37^{\circ} \mathrm{C}$. The aorta was then excised and placed into serum-free DMEM overnight at $37^{\circ} \mathrm{C}$. The next day, aortae were placed into Krebs-Henseleit bicarbonate buffer solution (KHS). The composition of KHS was $118.3 \mathrm{mmol} / \mathrm{L} \mathrm{NaCl}, 4.7 \mathrm{mmol} / \mathrm{L} \mathrm{KCl}, 2.5$ $\mathrm{mmol} / \mathrm{L} \mathrm{CaCl} 2,1.2 \mathrm{mmol} / \mathrm{L} \mathrm{MgSO} 4,1.2 \mathrm{mmol} / \mathrm{L}$ $\mathrm{KH}_{2} \mathrm{PO}_{4}, 25 \mathrm{mmol} / \mathrm{L} \mathrm{NaHCO}_{3}, 5.6 \mathrm{mmol} / \mathrm{L}$ glucose, plus $0.01 \mathrm{mmol} / \mathrm{L}$ ibuprofen, gassed with $5 \% \mathrm{CO}_{2}$ to 
maintain a $\mathrm{pH}$ of 7.4 at $37^{\circ} \mathrm{C}$. Adventitial fat was carefully removed, and arteries were cut into rings (2-mm thickness). The rings were suspended by two tungsten wires $(25-\mu \mathrm{m}$ diameter) and mounted with $1.5 \mathrm{~g}$ of resting tension in a vessel myograph system $(5-\mathrm{mL}$ chamber size; Kent Scientific, Litchfield, Connecticut, USA). Preliminary experiments demonstrated that this base-line tension resulted in optimal length-tension curves. Isometric tension was measured using a force transducer coupled to a data acquisition system. After an equilibration period of 60 minutes, rings were precontracted with phenylephrine $(1 \mu \mathrm{M})$ until a plateau was reached. Vessels were then washed, and this was repeated at least three times in order to stabilize the tissue. In experiments examining vasorelaxation, rings were pre-contracted with phenylephrine, and responses to the Ach $\left(1 \times 10^{-9}\right.$ to $\left.5 \times 10^{-5} \mathrm{M}\right)$ and sodium nitroprusside (SNP; $1 \times 10^{-9}$ to $5 \times 10^{-5} \mathrm{M}$ ) were quantified.

Detection of $\beta$-gal expression. Vessel segments, harvested 3 days after surgery or in vitro transduction with Adeno$\beta$-gal transduction, were washed with cold PBS and fixed for 10 minutes in $1 \%$ paraformaldehyde. $\beta$-gal activity was assessed by incubating arteries in 5-bromo4-chloro-3-indolyl- $\beta$-D-galactopyranoside (X-gal) chromagen as described previously (16). The vessels were cut longitudinally and photographed through a dissecting microscope for macroscopic assessment. The specimens were then embedded in paraffin for histologic evaluation. Five-millimeter cross-sections were counterstained with hematoxylin and eosin.

Statistical methods. Data were evaluated with a twotailed, unpaired Student's $t$ test, a one-way ANOVA followed by a Fisher $t$-test, or a two-way ANOVA, and expressed as the mean \pm SEM. A value of $P<0.05$ was considered statistically significant (NS = not significant).

\section{Results}

To assess the role of Akt in regulating vasomotor responses, a rabbit femoral artery model of adenovirusmediated Akt gene transfer to vascular endothelium was established (Figure 1a). This procedure involved temporary ligation of femoral artery, popliteal and saphenous arteries, and infusion of the isolated femoral artery with adenoviral solutions via the saphenous artery. After a 15minute incubation, temporary clamps on the proximal femoral artery and popliteal arteries were removed, and the saphenous artery was permanently ligated (Figure 1b). Three days after infection or mock infection, vessels were assessed for transgene expression or vasomotor responses and blood flow.

Transgene expression was assessed in femoral arteries infected with Adeno- $\beta$-gal. Vessels were harvested at 3 days after infection and stained with X-gal. En face analysis of opened vessels revealed greater than $50 \%$
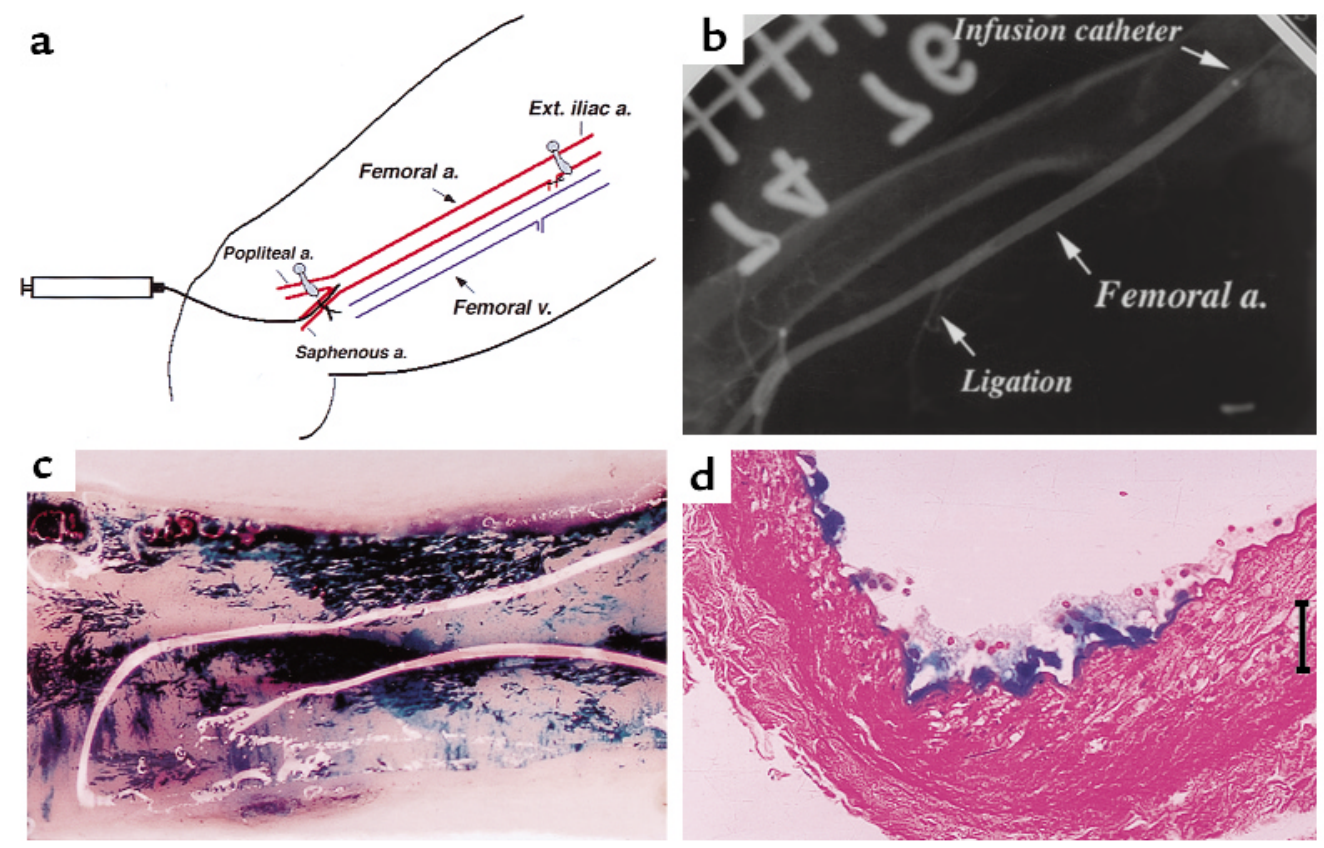

\section{Figure 1}

Rabbit femoral artery model of adenovirus-mediated gene transfer. (a) Femoral arteries were temporarily isolated by clamping the proximal femoral, popliteal, and saphenous arteries. Solutions of saline alone or saline containing adenoviral constructs that express $\beta$-gal, dn-Akt, or myr-Akt were then infused through the saphenous artery. After incubation for 15 minutes, the saphenous artery was permanently ligated and the temporary clamps on the femoral and popliteal arteries were removed. (b) Vessel diameter was assessed angiographically at 3 days after treatment, using an automated edge-detection system. The site of permanent saphenous artery ligation, after adenovirus administration, is shown. (c) Specific and efficient adenovirus-mediated gene transfer to the endothelium. Four rabbit femoral arteries were treated with an adenoviral construct expressing $\beta$-gal and harvested after 3 days. $\beta$-gal expression detected by X-gal staining from a representative en face view is shown. (d) Cross section of vessel demonstrating that $\beta$-gal expression is detected in the endothelium, but not in medial smooth muscle cells. Bar: $50 \mu \mathrm{m}$. v., vein; ext., external; a., artery. 


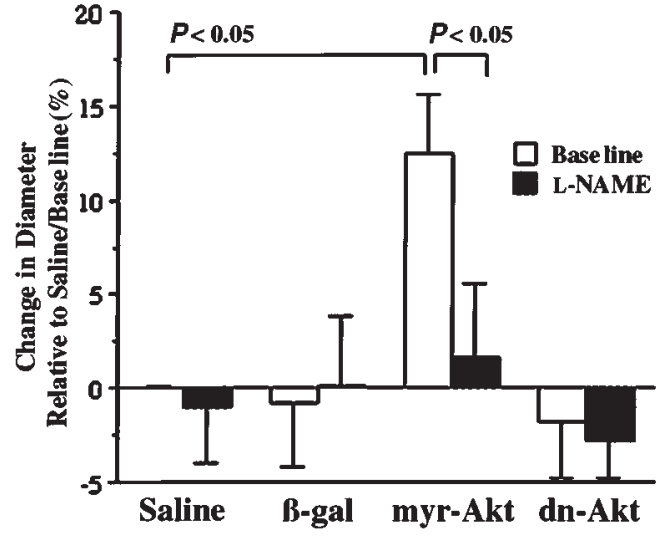

Figure 2

Constitutive activation of Akt promotes vasodilation that is reversed by infusion of L-NAME. Vessel diameter was determined by quantitative angiography 3 days after endothelial infection. Data are expressed as the percentage change in vessel diameter relative to the base-line (untreated) diameter in the mock-infected (saline) group. Data are expressed as mean $\pm \operatorname{SEM}(n=5)$.

transduction. A representative X-gal-stained vessel is shown in Figure 1c. Furthermore, transgene expression was exclusively localized in the endothelium, and no expression was detected in medial smooth muscle cells, as determined by analyses of vessel cross sections (Figure 1d). No X-gal-positive cells could be detected in non-treated vessels (data not shown).

To determine the consequences of transgene expression on vasomotor responses, quantitative angiography was performed on femoral vessels incubated with saline alone (mock infected) or with adenoviral constructs expressing $\beta$-gal, constitutively active Akt (myr-Akt), or dn-Akt. At 3 days after treatment, base-line vessel diameter was as follows: saline-treated, $1.23 \pm 0.04 \mathrm{~mm} ; \beta$ gal, $1.20 \pm 0.06 \mathrm{~mm}(P=\mathrm{NS})$; dn-Akt, $1.17 \pm 0.06 \mathrm{~mm}$ $(P=\mathrm{NS})$; and myr-Akt, $1.38 \pm 0.02 \mathrm{~mm}(P<0.05)$. Thus, vessels infected with myr-Akt displayed significantly larger base-line diameters than mock-infected (saline) or $\beta$-gal-transduced vessels, whereas transduction with dn-Akt did not significantly affect base-line vessel diameter. To test whether myr-Akt promotes vasodilation through an NO-dependent mechanism, vessels were infused with L-NAME, an L-arginine analog that specifically inhibits eNOS. Infusion of L-NAME diminished vessel diameter in the myr-Akt-treated group to

\section{Table 1}

Papavarine and nitroglycerin effects on femoral artery flow and diameter

$\begin{array}{llcc}\text { Transduction } & \text { Treatment } & \text { Diameter }(\mathrm{mm}) & \text { Flow }(\mathrm{mL} / \mathrm{min}) \\ \text { Mock } & \text { Saline } & 1.23 \pm 0.02 & 7.1 \pm 0.3 \\ \text { Mock } & \text { Papavarine } & 1.26 \pm 0.02 & 15.9 \pm 0.8^{\mathrm{A}} \\ \text { Mock } & \text { NTG } & 1.40 \pm 0.07^{\mathrm{A}} & 13.5 \pm 1.2^{\mathrm{A}} \\ \text { dn-Akt } & \text { Saline } & 1.20 \pm 0.04 & 6.8 \pm 0.4 \\ \text { dn-Akt } & \text { Papavarine } & 1.24 \pm 0.01 & 15.3 \pm 0.7^{\mathrm{A}}\end{array}$

${ }^{A} P<0.05$ relative to the saline-treatment group. a size that was comparable to the base line, saline-treated group (Figure 2). In contrast, L-NAME had no significant effect on the base-line diameters of saline or of $\beta$-gal- or dn-Akt-transduced vessels.

To assess further whether Akt gene transfer modulates $\mathrm{NO}$ production in the vessel wall in vivo, we assayed cyclic GMP accumulation as a bioassay for NO synthesis. At 3 days after infection, Adeno- $\beta$-gal-treated femoral vessels contained $174 \pm 25 \mathrm{fmol} \mathrm{cGMP} / \mathrm{mg}$ protein, consistent with basal cGMP levels reported by others (19). Infection of vessels with adenoviral constructs expressing myr-Akt increased cGMP levels to $244 \pm 25$ $\mathrm{fmol} / \mathrm{mg}$ protein $(P<0.05)$, consistent with an elevation of NO in the vessel, whereas dn-Akt-treated vessels contained $144 \pm 26 \mathrm{fmol} \mathrm{cGMP} / \mathrm{mg}$ protein $(P=\mathrm{NS})$.

Vasomotor responses to acetylcholine (Ach), an endothelium-dependent (NO-mediated) vasodilator, and NTG, an endothelium-independent vasodilator that generates $\mathrm{NO}$, were also assessed by quantitative angiography (Figure 3). Expression of dn-Akt in the endothelium abolished Ach-induced changes in diameter without altering the vasodilatory response to NTG, consistent with an inhibition of endothelium-derived NO. Infection with adenoviral constructs expressing $\beta$ gal or myr-Akt did not significantly influence maximal vessel diameter attained after the infusion of Ach or NTG relative to mock-infected (saline) vessels.

Blood flow through treated femoral arteries was assessed by intravascular Doppler analysis (Figure 4). Under base-line conditions, myr-Akt-transduced vessels displayed a significant increase in resting blood flow relative to saline-, $\beta$-gal- or dn-Akt-treated vessels consistent with the ability of myr-Akt to increase resting vessel diameter. Accordingly, the relative Ach- or NTG-induced changes in blood flow in the myrAkt-transduced vessels were less than that seen in

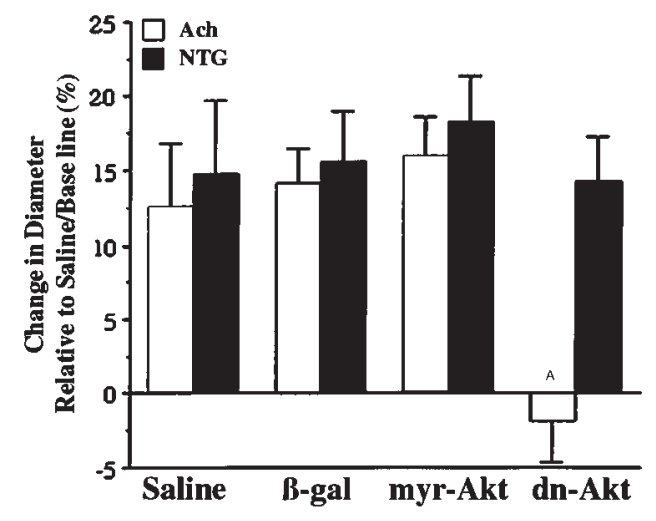

Figure 3

$\mathrm{dn}$-Akt blocks vasodilation in response to the endothelium-dependent agonist Ach, but vessels respond normally to NTG. Data are expressed as the percentage change in vessel diameter relative to the base-line (untreated) diameter in the mock-infected (saline) group as determined by quantitative angiography. Data are presented as mean \pm SEM. $\left(n=5\right.$; ${ }^{A} P<0.05$ relative to saline-treated, Adeno- $\beta$ gal-treated, or Adeno-myr-Akt-treated vessels in the presence of Ach, or all treatment conditions in the presence of NTG.) 


\section{Figure 4}

Assessment of blood flow in treated rabbit femoral arteries by Doppler guide wire measurements. Base-line blood flow is increased by endothelial expression of myr-Akt. Increased blood flow resulting from infusion of Ach is attenuated by endothelial expression of dnAkt, but vessels respond normally to NTG. Data are presented as mean \pm SEM. $\left(n=5\right.$; ${ }^{A} P<0.05$ relative to the same agonist treatment group in saline or Adeno- $\beta$-gal-treated vessels.) saline-treated or $\beta$-gal-transduced vessels, analogous to the previous data measuring Ach- and NTG-induced changes in vessel diameter. Importantly, the vasodilatory response to Ach was essentially abolished in dnAkt-transduced vessels compared with control groups, whereas responses to NTG were virtually identical to those seen in the control groups.

Rabbit femoral arteries were treated with papavarine to examine the relationship between flow and vasomotor responses. Consistent with the findings of a prior study (20), papavarine infusion led to a marked increase in flow through the femoral artery, yet had little or no effect on femoral artery diameter (Table 1), suggesting an effect on downstream arterioles. In contrast, treatment with NTG led to increases in both flow and vessel diameter. Transduction of vessels with dn-Akt had no effect on vessel diameter under these conditions, nor did it affect the papavarine-induced increase in flow.

To examine the role of Akt in agonist-induced vasomotion in greater detail, the endothelium of mouse aortae were transduced with dn-Akt or $\beta$-gal and explanted rings studied ex vivo. Mouse aortae were examined in these experiments because Ach induced vasorelaxation in this vessel is exclusively $\mathrm{NO}$ depend-

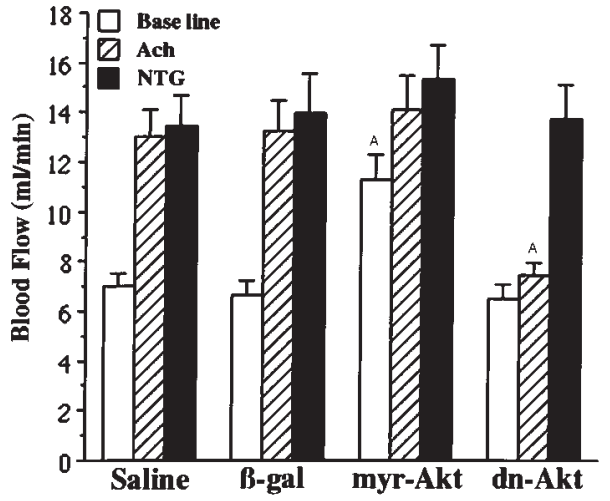

ent, as demonstrated by the loss of Ach-induced relaxations in eNOS knockout mice (21). Luminal adenoviral infection of the mouse aorta resulted in $50-60 \%$ transduction of the vessel wall (Figure 5a) that was confined to the vascular endothelium (Figure $5 b$ ). Interestingly, Ach-induced relaxations were significantly inhibited in the dn-Akt-transduced arteries relative to Adeno- $\beta$-gal-transduced vessel segments (Figure 5c). Transduction with dn-Akt shifted the Ach doseresponse curve rightward relative to control vessels with $\mathrm{EC}_{50}$ values of 64 versus $90 \mathrm{nM}$ for $\beta$-gal- and dnAkt-transduced vessels, respectively. In contrast, endothelium-independent relaxations to SNP were not different between the two groups (Figure $5 \mathrm{~d}$ ), with $\mathrm{EC}_{50}$ values of 106 versus $109 \mathrm{nM}$ for $\beta$-gal- and dnAkt-transduced vessels, respectively.

\section{Discussion}

This study documents a new physiological role for Akt signal transduction; the regulation of vasomotor tone. We show that enhanced activity of Akt in the endothelium increases the resting diameter of rabbit femoral arteries with functional consequences on blood flow. The results of this study are consistent with the in vitro

\section{Figure 5}

$\mathrm{dn}$-Akt attenuates aortic relaxations ex vivo in response to the endothelium-dependent agonist Ach, but not in response to SNP. Mouse aortae were cannulated and infused with adenoviral constructs that express either $\beta$ gal or dn-Akt, and vessels were placed into culture overnight. (a) Specific and efficient adenovirus-mediated gene transfer to the endothelium as determined by $\beta$-gal expression detected by $X$-gal staining from a representative en face view. The arrow indicates the direction of flow. (b) Cross section of vessel demonstrating that $\beta$-gal expression is detected in the endothelium, but not in medial smooth muscle cells or adventitia (A). L, lumen. Parts $\mathbf{a}$ and $\mathbf{b}$ are representative of three aortae. Bars represent 250 and $50 \mu \mathrm{m}$ in $\mathbf{a}$ and $\mathbf{b}$, respectively. Transduction of dn-Akt inhibits Ach-induced vascular relaxations (c), whereas SNP-induced relaxations are not affected (d). Data are presented as mean \pm SEM. ( $n=9$ rings for $\beta$-gal-transduced and 10 rings for $\mathrm{dn}$-Akt-transduced vessels isolated from four mice. ${ }^{A} P<0.05$ relative to the $\beta$-gal-transduced vessels by ANOVA.)
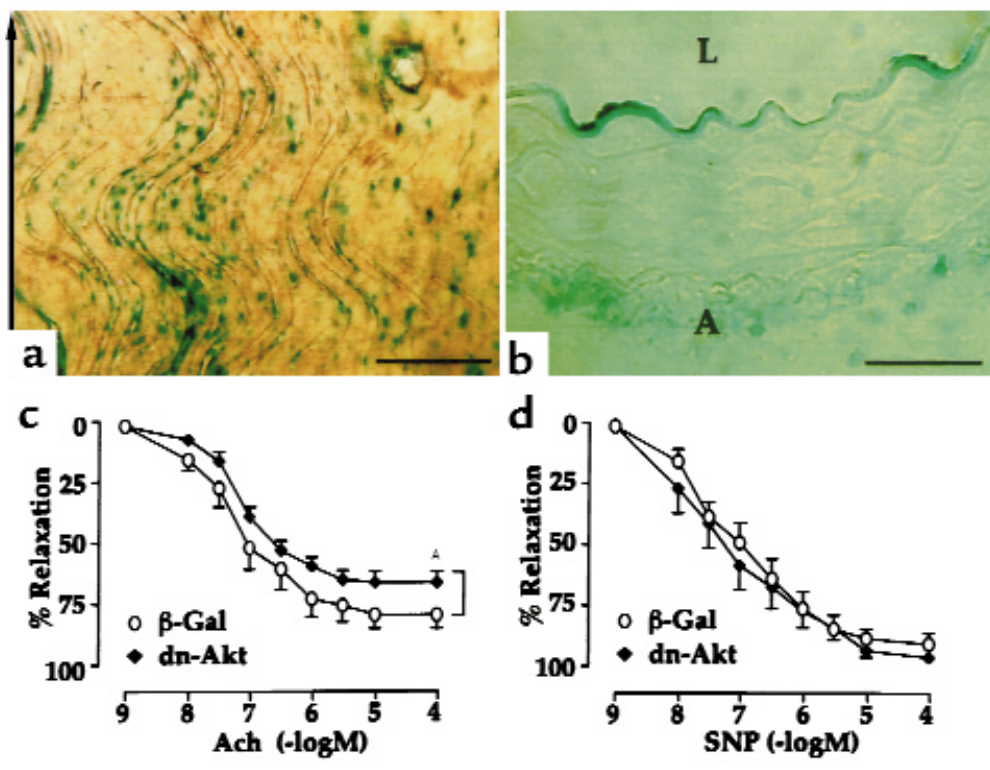
observations that Akt can directly phosphorylate eNOS leading to the activation of the enzyme and NO production in cultured bovine lung microvascular and human umbilical vein endothelial cells $(12,13)$. Consistent with an NO-dependent mechanism in vivo, the eNOS inhibitor L-NAME blocked vasodilation induced by constitutively active Akt gene transfer to the endothelium of rabbit femoral arteries. Also consistent with an eNOS-dependent pathway is the observation that cGMP levels, a downstream effector of $\mathrm{NO}$ in smooth muscle cells, are elevated in vessels in which myr-Akt is expressed in the endothelium.

We also show that dn-Akt gene transfer markedly blocked vasodilation in response to Ach, an endothelium-dependent agonist that mobilizes intracellular calcium and stimulates NO production (22). In vivo, Ach will trigger both local and systemic vasodilation, thereby increasing blood flow and the attendant shear stress through large vessels. This suggests that the inhibitory effect of dn-Akt on Ach-induced relaxation of the femoral artery may either be mediated by blocking the local vasodilation actions of this agent or by diminishing shear stress-induced NO release that is brought about by systemic vasodilation. To examine directly whether dn-Akt could influence local endotheliumdependent responses to Ach in the absence of a change in hemodynamics, mouse aortic endothelium was transduced with dn-Akt, and ring segments were examined ex vivo. In this model, dn-Akt inhibited the direct vasorelaxant response to Ach, but not SNP. Collectively, these experiments are consistent with the hypothesis that Ach-induced vasodilation is mediated by Akt phosphorylation of eNOS, and that Akt is likely to be involved in eNOS activation by calcium mobilizing agonists, such as Ach and VEGF $(12,23,24)$.

Interestingly, dn-Akt by itself did not produce statistically significant vasoconstriction as assessed by angiography, nor did it diminish blood flow as assessed by intravascular Doppler flow measurements. Furthermore, treatment with L-NAME also did not lead to a significant reduction in vessel diameter in control vessels, though it effectively blocked vasodilation in response to infection with Adeno-myr-Akt. These data suggest that within the untreated rabbit femoral artery, a conduit vessel lacking myogenic tone, the Akt-eNOS regulatory circuit does not significantly contribute to vessel diameter under conditions of resting flow. Furthermore, intra-arterial injection of papavarine was found to increase flow through this segment markedly, but it did not increase vessel diameter. Likewise, the papavarineinduced flow was unaffected by transduction with dnAkt. Collectively, these data indicate that there is minimal Akt-mediated NO release in response to flow and the attendant increase in shear stress in this model.

The effects of acute Akt gene delivery on vasomotor tone are comparable to the consequences of eNOS overexpression in transgenic mice. It is reported that chronic eNOS expression by the endothelium results in increased basal cGMP production by the vessel and hypotension that can be reversed by L-NAME administration (25). Furthermore, adenovirus-mediated transfer of the eNOS gene in normal or atherosclerotic rabbit arteries diminishes contractile responses and enhances endothelium-dependent relaxation in vitro (26-28). To our knowledge, the data herein are the first demonstration that acute gene transfer can modulate vasomotor responses in vivo, and they show that Akt functions as a physiological regulator of $\mathrm{NO}$ production in the vessel wall. In addition to controlling vasomotor tone, $\mathrm{NO}$ production by the endothelium has important protective functions: NO promotes endothelial cell survival (29), inhibits platelet adhesion (30), reduces inflammatory cell infiltration (31), and inhibits smooth muscle cell proliferation $(32,33)$. Recognition that Akt is a regulator of eNOS in the intact animal suggests that impairment of endothelial cell function and NO synthesis, as occurs in hypercholesterolemia and atherosclerosis $(34,35)$, may result from perturbations in the Akt-eNOS regulatory interaction. Hence, these data indicate that the pharmacologic modulation of Akt activity could have utility for the treatment of vascular disorders associated with endothelial cell dysfunction and impaired blood flow.

\section{Acknowledgments}

This work was supported by NIH grants AG-15052, AR-40197, and HL-50692 (to K. Walsh); HL-51948, HL-50974, and HL-64793 (to W.C. Sessa); and F32 HL10183 (to D. Fulton). W.C. Sessa is an Established Investigator of the American Heart Association.

1. Moncada, S., Palmer, R.M.J., and Higgs, E.A. 1991. Nitric oxide: physiology, pathophysiology and pharmacology. Pharmacol. Rev. 43:109-142.

2. Garcia-Cardena, G., et al. 1998. Dynamic activation of endothelial nitric oxide synthase by Hsp90. Nature. 392:821-824.

3. Garcia-Cardena, G., Fan, R., Stern, D.F., Liu, J., and Sessa, W.C. 1996. Endothelial nitric oxide synthase is regulated by tyrosine phosphorylation and interacts with caveolin-1. J. Biol. Chem. 271:27237-27240.

4. Corson, M.A., et al. 1996. Phosphorylation of endothelial nitric oxide synthase in response to fluid shear stress. Circ. Res. 79:984-991.

5. Coffer, P.J., Jin, J., and Woodgett, J.R. 1998. Protein kinase B (c-Akt): a multifunctional mediator of phosphatidylinositol 3-kinase activation. Biochem. J. 335:1-13.

6. Fujio, Y., Nguyen, T., Wencker, D., Kitsis, R.N., and Walsh, K. 2000. Akt promotes survival of cardiomyocytes in vitro and protects against ischemia-reperfusion injury in mouse heart. Circulation. 101:660-667.

7. Dudek, H., et al. 1997. Regulation of neuronal survival by the serinethreonine protein kinase Akt. Science. 275:661-665.

8. Kauffmann-Zeh, A., et al. 1997. Suppression of c-Myc-induced apoptosis by Ras signalling through PI(3)K and PKB. Nature. 385:544-548.

9. Kennedy, S.G., et al. 1997. The PI 3-kinase/Akt signaling pathway delivers an anti-apoptotic signal. Genes Dev. 11:710-713.

10. Fujio, Y., and Walsh, K. 1999. Akt mediates cytoprotection of endothelial cells by vascular endothelial growth factor in an anchorage-dependent manner. J. Biol. Chem. 274:16349-16354.

11. Gerber, H.-P., et al. 1998. Vascular endothelial growth factor regulates endothelial cell survival through the phosphatidylinositol 3'kinase/Akt signal transduction pathway: requirement for Flk-1/KDR activation. J. Biol. Chem. 273:30336-30343.

12. Fulton, D., et al. 1999. Regulation of endothelium-derived nitric oxide production by the protein kinase Akt. Nature. 399:597-601.

13. Dimmeler, S., et al. 1999. Activation of nitric oxide synthase in endothelial cells by Akt-dependent phosphorylation. Nature. 399:601-605.

14. Fujio, Y., et al. 1999. Cell cycle withdrawal promotes myogenic induction of Akt, a positive modulator of myocyte survival. Mol. Cell. Biol. 19:5073-5082. 
15. Smith, R.C., et al. 1997. p21 $1^{\text {CIP1 }}$-mediated inhibition of cell proliferation by overexpression of the gax homeodomain gene. Genes Dev. 11:1674-1689.

16. Rivard, A., et al. 1999. Early cell loss after angioplasty results in a disproportionate decrease in percutaneous gene transfer to the vessel wall. Hum. Gene Ther. 10:711-721.

17. Doucette, J.W., et al. 1992. Validation of a Doppler wire for intravascular measurements of coronary artery flow velocity. Circulation. 85:1899-1911.

18. Arnold, W., Mittal, C., Katsuki, S., and Murad, F. 1977. Nitric oxide activates guanylate cyclase and increases guanosine $3^{\prime}: 5^{\prime}$-cyclic monophosphate levels in various tissue preparations. Proc. Natl. Acad. Sci. USA. 74:3203-3207.

19. Martin, W., Villani, G.M., Jothianandan, D., and Furchgott, R.F. 1985. Selective blockade of endothelium-dependent and glyceryl trinitrateinduced relaxation by hemoglobin and by methylene blue in the rabbit aorta. J. Pharmacol. Exp. Ther. 232:708-716.

20. Bauters, C., et al. 1994. Physiological assessment of augmented vascularity induced by VEGF in ischemic rabbit hindlimb. Am. J. Physiol. 267:H1263-H1271.

21. Huang, P.L., et al. 1995. Hypertension in mice lacking the gene for endothelial nitric oxide synthase. Nature. 377:239-242.

22. Singer, H., and Peach, M. 1982. Calcium- and endothelial-mediated vascular smooth muscle relaxation in rabbit aorta. Hypertension. 3:19-25.

23. Papapetropoulos, A., Garcia-Cardena, G., Madri, J.A., and Sessa, W.C. 1997. Nitric oxide production contributes to the angiogenic properties of vascular endothelial growth factor in human endothelial cells. $J$. Clin. Invest. 100:3131-3139.

24. Michell, B.J., et al. 1999. The Akt kinase signals directly to endothelial nitric oxide synthase. Curr. Biol. 9:845-848.

25. Ohashi, Y., et al. 1998. Hypotension and reduced nitric oxide-elicited vasorelaxation in transgenic mice overexpressing endothelial nitric oxide synthase. J. Clin. Invest. 102:2061-2071.
26. Ooboshi, H., Toyoda, K., Faraci, F.M., Lang, M.G., and Heistad, D.D. 1998. Improvement of relaxation in an atherosclerotic artery by gene transfer of endothelial nitric oxide synthase. Arterioscler. Thromb. Vasc. Biol. 18:1752-1758.

27. Kullo, I.J., et al. 1997. Enhanced endothelium-dependent relaxations after gene transfer of recombinant endothelial nitric oxide synthase to rabbit carotid arteries. Hypertension. 30:314-320.

28. Channon, K.M., et al. 1998. In vivo gene transfer of nitric oxide synthase enhances vasomotor function in carotid arteries from normal and cholesterol-fed rabbits. Circulation. 98:1905-1911.

29. Dimmeler, S., Haendeler, J., Nehls, M., and Zeiher, A.M. 1997. Suppression of apoptosis by nitric oxide via inhibition of interleukin$1 \beta$-converting enzyme (ICE)-like and cysteine protease protein (CPP)32-like proteases. J. Exp. Med. 185:601-607.

30. Simon, D.I., et al. 1993. Antiplatelet properties of protein S-nitrosothiols derived from nitric oxide and endothelium-derived relaxing factor. Arterioscler. Thromb. 13:791-799.

31. Qian, H.S., Neplioueva, V., Shetty, G.A., Channon, K.M., and George, S.E. 1999. Nitric oxide synthase gene therapy rapidly reduces adhesion molecule expression and inflammatory cell infiltration in carotid arteries of cholesterol-fed rabbits. Circulation. 99:2979-2982.

32. von der Leyen, H.E., et al. 1995. Gene therapy inhibiting neointimal vascular lesion: in vivo transfer of endothelial cell nitric oxide synthase gene. Proc. Natl. Acad. Sci. USA. 92:1137-1141.

33. Guo, K., Andrés, V., and Walsh, K. 1998. Nitric oxide-induced downregulation of cdk2 activity and cyclin A gene transcription in vascular smooth muscle cells. Circulation. 20:2066-2072.

34. Heistad, D.D., Armstrong, M.L., Marcus, M.L., Piegors, D.J., and Mark, A.L. 1984. Augmented responses to vasoconstrictor stimuli in hypercholesterolemic and atherosclerotic monkeys. Circ. Res. 54:711-718.

35. McLenachan, J.M., Williams, J.K., Fisher, R.D., Ganz, P., and Selwyn, A.P. 1991. Loss of flow-mediated endothelium-dependent dilation occurs early in the development of atherosclerosis. Circulation. 84:1273-1278. 